\title{
Spectrum allocation algorithm under the novel channel handover constraint in cognitive radio network
}

\author{
Kuangyu Chen ${ }^{1}$, Fanzi Zeng ${ }^{2}$, Qingguang Zeng \\ College of information science and engineering, Hunan University \\ Changsha, China \\ 1e-mail:kuangyuchen0110@126.com, ${ }^{2}$ e-mail:zengfanzi @ 126.com
}

\begin{abstract}
Dynamic spectrum (channel) allocation in cognitive radio network is the effective measure to solve the low utility of spectrum resource. Spectrum (channel) handover will degrade network performance and cause large amounts of energy consumption. This paper proposes a novel spectrum allocation algorithm to reduce the channel handover which allocates the channel that its conditional expectation is larger than the required slot that user's data need be completely transmitted once to user. We firstly model the channel as first-order two-state Markov process and achieve the channel statistical characteristic. Then we construct the spectrum allocation optimization with the interference constraints, channel availability constraint and channel handover constraint, which is solved by the colorsensitive graph coloring theory. Simulation results show that the proposed algorithm can more effectively reduce the number of channel handover of cognitive users compared to the CSGC algorithm and RDA algorithm and can achieve the satisfying system bandwidth and fairness.
\end{abstract}

Keywords-Cognitive radio; Markov process; spectrum allocation; channel handover; graph coloring theory

\section{INTRODUCTION}

Due to the enormous growth of wireless applications in recent years, wireless spectrum is getting more congested. Furthermore, the underutilization of the licensed spectrum bands makes the situation even worse ${ }^{[1]}$. Cognitive Radio $(\mathrm{CR})^{[2]}$ has been proposed as a fundamental solution to this problem. Cognitive radio techniques can improve spectrum efficiency by allowing secondary users to temporarily utilize the unused licensed spectrum of primary users in CR networks. Cognitive radios enable the users to communicate over the most appropriate spectrum bands through four main functionalities: spectrum sensing, spectrum management, spectrum mobility, and spectrum allocation.

There are already many researches which analyze the spectrum allocation algorithm in recent years. In [3-4], the authors solved the problem of low utilization of spectrum resources, but performance improvement was limited. In view of the spectrum allocation of efficiency and fairness problem, distributed greedy algorithm (DGA) and rand distributed algorithm (RDA) was proposed in [5]. In [6-7], color-sensitive Graph Coloring (CSGC) was proposed based on above graph-coloring theory whose main idea was considering the SINR of different users on every channel. The better the channel condition is, the greater the weight of channel will be. In [8-9], the authors provided game theoretical analyses in cognitive radio networks for distributed channel allocation. In [10], the author proposed a Markov random field based framework for CR networks with the aim of increasing spectrum utilization by exploiting missing channel opportunities. In [11], a hidden Markov model based spectrum predictor was proposed.

However, most of the related works don't consider reducing the number of channel handover problem, but channel handover can increase the node energy consumption. Lots of experimental results show that the channel handover cost energy for $110.75 \%$ of the energy required for transmission. And frequent spectrum handover can make the whole system performance degradation ${ }^{[12]}$ Therefore, for energy constrained cognitive wireless network, when design the channel allocation algorithm, need to reduce the handover times as much as possible. In this paper, we choose Markov model to predict channel state and achieve the channel characteristic. Based on channel characteristic we propose a novel algorithm which allocates the channels whose characteristic is best match to node's data size. The algorithm not only decrease the number of channel handover of system, but also maintain high spectrum utilization and fairness.

The rest of the paper is organized as follows. In Section II, we described problem model and problem. In Section III, we proposed the spectrum allocation algorithm. In Section V, we provide the simulations for the algorithm and discussion. Finally, Section VI concludes this paper.

\section{CHANNEL MOdEL AND PROBLEM FORMULATION SELECTING A TEMPLATE}

Suppose there are $\mathrm{N}$ cognitive users in a cognitive radio network sharing $K$ orthogonal primary channels. Assuming that each of primary channels only have two states: busy or idle, and is modeled as the first-order two-state Markov process illustrated in fig. 1. The busy state means that primary channel is occupied by primary user and not available for the CR users; the idle state represents that this primary channel is available for the CR users. In Figure 1, $p_{j}$ is the transition probability the channel $j$ from idle to busy; $q_{j}$ is the transition probability of the channel $j$ from busy to idle. 


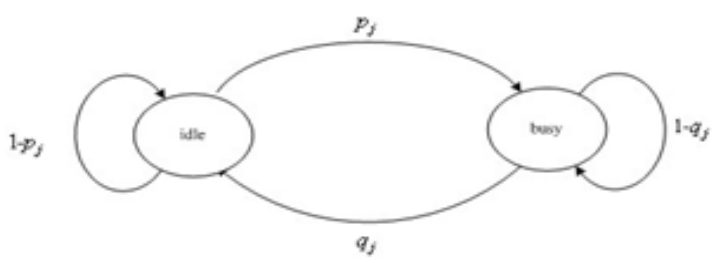

Figure 1. channel state model.

According to the first-order two-state Markov process, the probability that channel is in busy state is as follows:

$$
P_{k}^{\text {busy }}=\frac{p_{k}}{p_{k}+q_{k}}
$$

If the channel $k$ is idle at slot $\mathrm{t}$, the probability that this channel is idle for the next $L$ slots is $\left(1-p_{k}^{\text {busy }}\right)^{L}$. Then the expectation of the length of slots where channel $k$ is successively idle is as follows:

$$
E_{k}(L)=\sum_{L} L\left(1-p_{k}^{\text {busy }}\right)^{L}
$$

Let $r_{k}$ denote the rate that the channel $k$ can support, $d_{j}$ represent the data size that CR user $j$ need to transmit. $\theta_{k, j}$ represent the number of slots required by $\mathrm{CR}$ user $j$ finishing transmission of $d_{j}$ on channel $k$ one time. $\theta_{k, j}$ can be calculated as follows:

$$
\theta_{k, j}=d_{j} / r_{k}
$$

When dealing with the channel allocation problem, CR user $j$ should choose the channel $k$ that $\theta_{k, j}$ is smaller or equal to $E_{k}(L)$, in this case CR user $j$ is more likely to finish its data transmission without channel handover.

With the channel model and the expectation of the length of slots in mind, now we turn to address the channel allocation problem, and introduce the following notation.

1) Channel availability

$$
L=\left\{l_{n, k} \mid l_{n, k} \in\{0,1\}, n=1, \cdots, N ; k=1, \cdots, K\right\}_{N \times K},
$$

which represents the channel availability. $l_{n, k}=1$ means that channel $k$ is available to user $n$.

2) Interference constraint

$C=\left\{c_{i, j, k} \mid c_{i, j, k} \in\{0,1\}\right\}_{N \times N \times K}$, which represents the interference constraint between cognitive users. If $c_{i, j, k}=1$, user $i$ and $j$ would interfere with each other once they use channel simultaneously.

3) Channel reward

$B=\left\{b_{n, k} \mid n=1, \cdots, N ; k=1, \cdots, K\right\}_{N \times K}$, which represents the channel reward. $b_{n, k}$ represents maximum throughput that can be acquired by user $n$ using channel $k$.

4) Channel allocation

$$
D=\left\{d_{n, k} \mid d_{n, k} \in\{0,1\}, n=1, \cdots, N ; k=1, \cdots, K\right\}_{N \times K} \quad,
$$

$d_{n, k}=1$ represents channel $k$ is allocated to user $n$.

5) Initial channel allocation matrix

$A=\left\{a_{n, k} \mid a_{n, k} \in\{0,1\}\right\}_{N \times k}$.Channel is allocated only when it is available, so the constraint need to be satisfied, $d_{n, k} \leq l_{n, k}$

Moreover, one channel can't be allocated simultaneously to users who will interfere with each other. This constraint can be formulated mathematically as follows:

$$
d_{i, k} d_{j, k} c_{i, j, k}=0, \forall i, j=1, \cdots, N ; k=1, \cdots, K
$$

As stated above, when dealing with the channel allocation problem, CR user $j$ should choose the channel $k$ that $\theta_{k, j}$ is smaller or equal to $E_{k}(L)$. This can be formulated :

$$
0<d_{n, k} \theta_{k, j} \leq E_{k}
$$

Let $h_{n, k}$ represent the handover of $n$ user on channel $k$. Thus we can calculate $h_{n, k}$ as follows:

$$
h_{n, k}=1-a_{n, k} d_{n, k}
$$

Then the total handovers $H$ in cognitive radio network can be calculated as follows:

$$
H=\sum_{n=1}^{N} \sum_{k=1}^{M} h_{n, k}
$$

When allocating channel, the total handovers $H$ should be minimized as to save the energy:

$$
\min H=\sum_{n=1}^{N} \sum_{k=1}^{M} h_{n, k}
$$

Except the objective (9), another objective for channel allocation algorithm is to maximize the channel allocation reward, this can be formulated as follows:

$$
\max \sum_{n=1}^{N} \sum_{k=1}^{K} d_{n, k} \cdot b_{n, k}
$$

Put (4), (5), (6), (8), (9), (10) together, the following global optimization problem was constructed as follows:

$$
\begin{aligned}
& \max \sum_{n=1}^{N} \sum_{k=1}^{K} d_{n, k} \cdot b_{n, k} \\
& \min H=\sum_{n=1}^{N} \sum_{k=1}^{K} h_{n, k} \\
& \text { s.t. } \quad \sum_{k=1}^{K} d_{n, k} \leq 1, d_{n, k} \leq l_{n, k} \\
& d_{i, k} d_{j, k} c_{i, j, k}=0, \forall i, j=1, \cdots, N ; k=1, \cdots, K \\
& 0<d_{j, k} \theta_{k, j} \leq E_{k}
\end{aligned}
$$

\section{IMPLEMENTATION OF CHANNEL ALLOCATION ALGORITHM}

The optimization problem (11) has two objectives, it is intractable. To solve the above problem, we need to do some modification and introduce an extra parameter $R$. 11(b) is formulated as constraint: $H \leq R$. Furthermore, if 
$d_{j, k} \theta_{k, j}>E_{k}$, CR user $j$ can not finish transmission on channel $k$, so need to make channel handover. Let $U(t)$ represent the step function. The handover from the mismatch between the data and the available slot of the chosen channel can be calculated as follows:

$$
H^{\prime}=\sum_{j=1, k=1}^{j=N, k=K} U\left(d_{j, k} \theta_{k, j}-E_{k}\right)
$$

The optimization problem (11) can be written as follows:

$$
\begin{aligned}
& \max \sum_{n=1}^{N} \sum_{k=1}^{K} d_{n, k} \cdot b_{n, k} \\
& \text { s.t. } \sum_{n=1}^{N} \sum_{k=1}^{K}\left(h_{n, k}+U\left(d_{j, k} \theta_{k, j}-E_{k}\right)\right) \leq R \\
& \sum_{k=1}^{K} d_{n, k} \leq 1, \quad d_{n, k} \leq l_{n, k} \\
& d_{i, k} d_{j, k} c_{i, j, k}=0, \forall i, j=1, \cdots, N ; k=1, \cdots, K
\end{aligned}
$$

The above channel allocation can be equivalent to graph vertex coloring problem where vertex represent cognitive users, colors represent channel, connection between vertices denote interference between the two cognitive users. To solve the above problem, we put forward an improving CSGC algorithm. Let:

$r_{n, k}=\frac{d_{j, k} \theta_{k, j}}{E_{k}}, \omega=h_{n, k}+U\left(r_{n, k}-\beta_{1}\right)-U\left(r_{n, k}-\beta_{2}\right)$,

where $\beta_{1}, \beta_{2}$ are associated with $R_{1}, R_{2}$. Thus we can get the label of node and corresponding color as follows:

$$
\begin{aligned}
& \text { label }_{n}=\max b_{n, k} \cdot \omega / g_{n, k}+1 \\
& \text { color } n_{n}=\arg \max ^{b_{n, k} \cdot \omega} / g_{n, k}+1
\end{aligned}
$$

Where label $_{n}$ is labeling value of user $n$, color ${ }_{n}$ is the channel selected by the user, $g_{n, k}$ mean the number of user can't use the same channel with user $n$ and is calculated as follows,

$$
g_{n, k}=\sum_{j=0, j \neq n}^{N-1} c(n, j, k) \cdot l_{j, k} \cdot l_{n, k}
$$

The steps of the improving CSGC algorithm are described as follows:

Step1: Initialize, set parameters and simulation model.

Step2: According to the labeling rules, calculate each vertex's (user) label $_{n}$ on each channel in its available channel list.

Step 3: Search the vertex that has the maximum label value. According to the maximum label value, allocate the corresponding color $_{n}$ (channel) to the vertex.

Step4: Update the topology and the matrixes. Delete the corresponding color from the available channel lists of the SU. Refresh the matrix value of $L, C, D, A$. Delete the vertexes with the empty color list and the associated edges.

Step5: If the user available channel matrix $L$ is empty, the allocation is finished, else jump to step2.

According to the latest matrix $D$ we can get the results of allocation. Including the total handovers $H$, we also introduce the total system bandwidth $U$ and the fairness index $^{[13]} \Gamma$ as performance metrics to evaluate the performance of the proposed algorithm.

$$
\begin{aligned}
& U=\sum_{n=1}^{N} \sum_{k=1}^{K} \boldsymbol{d}_{n, k} \cdot \boldsymbol{b}_{n, k} \\
& \Gamma_{\text {Faimess }}=\left\{\left(\sum_{n=1}^{N} \sum_{k=1}^{K} \boldsymbol{d}_{n, k} \cdot \boldsymbol{b}_{n, k}\right)^{2} N \sum_{n=1}^{N}\left(\sum_{k=1}^{K} \boldsymbol{d}_{n, k} \cdot \boldsymbol{b}_{n, k}\right)^{2}\right\}
\end{aligned}
$$

Value of $\Gamma$ is the more close to 1 , the bandwidth users acquired is the more close to equal, fairness performance of algorithm is the better.

\section{THE SimULATION AND ANALYSIS}

This paper uses MATLAB software to simulate the algorithms. We conduct our simulations under the assumption of a noiseless, immobile radio network and randomly place a number of primary and secondary users in a given area $(10 \times 10)$. Each primary user randomly selects one channel to utilize from a pool of channels. Every cognitive user has the same disturbance radius. Simulation experiments are conducted 400 times, the average value as the final test results.

At first we investigate the handover performance of three algorithms. The number of available channels is set to 10 . The number of users is set randomly among 10-45. The simulation results of the three algorithms are as follows:

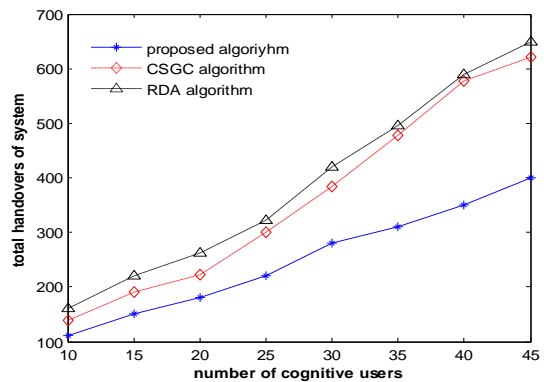

Figure 2. the comparison of channel switching of system

As shown in Fig. 2, the spectrum handover performance of the classical algorithm CSGC is relatively close to RDA. Compared with them, the spectrum handover performance of the proposed algorithm has the advantage apparently.

When the channel is set to 10 , we investigate the spectrum handover performance of three algorithms. The number of channel is set randomly among 10-24. The simulation results of the three algorithms are as follows:

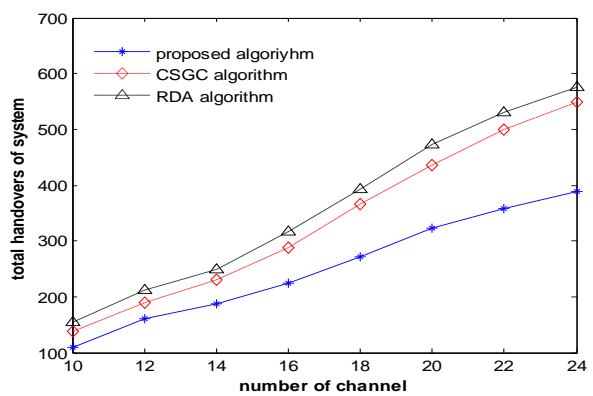

Figure 3. the comparison of channel switching of system. 
Fig.3 is the comparing simulation of CSGC algorithm, RDA algorithm and the proposed algorithm. The number of channel handover of three algorithm increases when the number of channels increases. The number of channel handover of the proposed algorithm is the least.

Then, we study the performance of the algorithms in total system bandwidth. The user number is set to 10.The number of channel is set randomly among 10-20. The simulation results of total system bandwidth as shown in Figure.4.

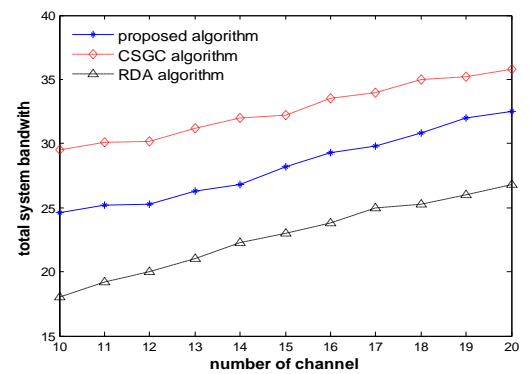

Figure 4. the comparison of channel system bandwidth

As shown in Fig. 4, with the increase in the number of channels, the total system bandwidth of the proposed algorithm increases, and it is better than RDA algorithm, but worse than CSGC algorithm.

In order to further verify the effectiveness of the algorithm, we investigated the performance of algorithms in the aspect of fairness. The user number is set to 30 . The number of users is set randomly among 20-40. The simulation results of the three algorithms are as follows:

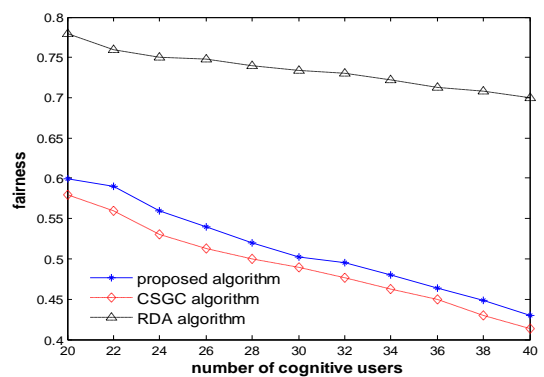

Figure 5. the comparison of the users' fairness

Fig. 5 shows the comparison of the users' fairness of the RDA algorithm, the CSGC algorithm and the proposed algorithm in this paper. All three algorithms degrade as the number of cognitive user increases. In addition, the RDA algorithm is the best and the proposed algorithm is better than the CSGC algorithm.

\section{CONCLUSION}

This paper firstly models the channel as first-order twostate Markov process and formulates the channel handover constraint between the conditional expectation of idle slot of channel and the required slots user finishes data transmission once. Then we construct the spectrum allocation optimization problem with the interference constraints, channel availability constraint and channel handover constraint. Finally we use the color-sensitive graph coloring theory to solve the spectrum allocation optimization problem. Because the channel handover constraint can assure channel allocated to user that can finish data-transmission on this channel. So it can more effectively reduce the channel handover compared to the CSGC algorithm and RDA algorithm and can achieve the satisfying system bandwidth and fairness. Simulation results validate the effectiveness of the proposed algorithm.

\section{ACKNOWLEDGMENT}

This work is supported in part by the National Natural Science Foundation of China under (No.61370096 and 61173012), the Key Project of Natural Science Foundation of Hunan Province under (No. 12JJA005) and technology project of Hunan Province (No.2013GK3023).

\section{REFERENCES}

[1] Hean Liu, Zhike Kuang, Cizhen Zhang, Goodson A. Yilly. Research on Spectrum Allocation Based on the Network Weights of Internet of Things. Journal of Networks, 2013, 8(12):2084-2811.

[2] Chouinard G, Lei Z D, Hu W D, et al. IEEE 802.22: the first cognitive radio wireless regional area network standard. IEEE Communications Magazine, 2009, 47(1):130- 138.

[3] Yang S M, Guo Wei. A cross-layer throughput-maximization algorithm for cognitive wireless Ad Hoc networks. Jisuanji Xuebao(Chinese Journal of Computers), 2012, 35(3): 491-503.

[4] Mange G, Rosik C, Leveil S. Cognitive resource management for QoS support in mobile opportunistic communications//Future Network \& Mobile Summit (FutureNetw), 2011. IEEE, 2011: 1-9.

[5] Wang W, Liu X. List-coloring based channel allocation for openspectrum wireless net works //Proc of the 62nd IEEE Vehicular Technology Conference (VTC). Dallas: IEEE, 2005: 690- 694.

[6] XIE Yu-peng, TAN Xue-zhi, LIU Yu-tao. A spectrum allocation algorithm based on graph theory. Applied Mechanics and Materials, 2012, 157: 1065-1070.

[7] WANG Jian-wu, ZHAO Qi, ZOU Jing-yuan. Advanced graphcoloring spectrum allocation algorithm for cognitive radio//Wireless Communications, Networking and Mobile Computing, 2009. WiCom'09. 5th International Conference on. IEEE, 2009: 1-4.

[8] Huang D, Chunyan Miao, Yuan Miao, Zhiqi Shen. A game theory approach for self-coexistence analysis among IEEE 802.22 networks//Information, Communications and Signal Processing, 2009. ICICS 2009. 7th International Conference on. IEEE, 2009: 1-5.

[9] Shweta Pandit, Ghanshyam Singh. Spectrum sharing in Cognitive Radio using game theory//Advance Computing Conference (IACC), 2013 IEEE 3rd International. IEEE, 2013: 1503-1506.

[10] E. Anifantis, V.Karyotis. A Markov Random Field framework for channel assignment in Cognitive Radio networks//Pervasive Computing and Communications Workshops (PERCOM Workshops), 2012 IEEE International Conference on. IEEE, 2012: 770-775.

[11] Tumuluru V K, Wang P, Niyato D. A neural network based spectrum prediction scheme for cognitive radio//Communications (ICC), 2010 IEEE International Conference on. IEEE, 2010: 1-5.

[12] [Wang L C, Wang C W, Feng K T. A queueing-theoretical framework for QoS-enhanced spectrum management in cognitive radio networks. Wireless Communications, IEEE, 2011, 18(6): 18-26.

[13] Ge Y, Sun J, Shao S. An improved spectrum allocation algorithm based on proportional fairness in Cognitive Radio networks//Communication Technology (ICCT), 2010 12th IEEE International Conference on. IEEE, 2010: 742-745 